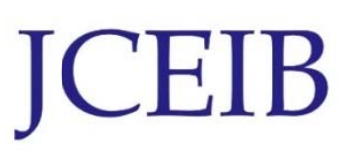

Journal Chemical Engineering and Industrial Biotechnology (JCEIB)

ISSN: 0126-8139 (Online); Open Access

Volume 5 Issue 5 pp. 74-78; May 2019

CUniversiti Malaysia Pahang Publisher

DOI: https://doi.org/10.15282/JCEIB-V5-07.29/3/2019/5.5

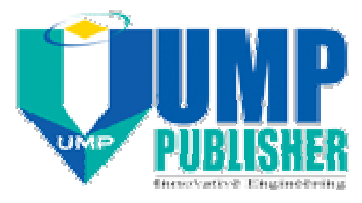

\title{
ELEMENTAL AND CHEMICAL COMPOSITION OF Moringa oleifera HUSK EXTRACTS
}

\author{
Mohammed Saedi Jami ${ }^{\mathrm{a}}$, Mani Malam Ahmad ${ }^{\mathrm{b} *}$, Olalere Olusegun Abayomi ${ }^{\mathrm{c}}$, Mohammed \\ Ngabura $^{\mathrm{d}}$ \\ ${ }^{\mathrm{a}}$ Department of Biotechnology Engineering, Faculty of Engineering, International Islamic University \\ Malaysia, Jalan Gombak, 53100 Kuala Lumpur, Malaysia. \\ ${ }^{b}$ Department of Biological Sciences, Faculty of Science, Kano University of Science and Technology, \\ Maiduguri Road, 3244, Wudil, Kano, Nigeria \\ 'Faculty of Chemical and Natural Resources Engineering, Universiti Malaysia Pahang, Lebuhraya Tun \\ Razak, 26300 Gambang, Kuantan, Pahang, Malaysia \\ ${ }^{\mathrm{d}}$ Department of Chemical and Environmental Engineering, Faculty of Engineering, Univeristi Putra \\ Malaysia, 43400 Serdang, Selangor, Malaysia \\ *Corresponding author:mmahmadu@gmail.com \\ Tel:+601136560301
}

\begin{abstract}
Moringa oleifera is a well-known tropical plant with myriads medicinal and nutritional benefits. Its nutraceutical functions are due to the presence of mineral element and phytochemical components which necessitate their profiling. In this study, the elemental and chemical profiles of husk wastes from Moringa oleifera plants were elucidated using Liquid Chromatography equipped with an electrospray time-of-flight mass spectrometer (LCMS-QToF) and X-ray Fluorescence analyser. The confirmation of 493 bioactive compounds and two higher concentrations of micronutrients (calcium and potassium) indicated the potential of the Moringa oleifera extracts for medicinal and nutritional purposes. Also, the minimal presence of trace elements such as $\mathrm{Cu}, \mathrm{Si}$, Mo and $\mathrm{Sr}$ showed that they are non-toxic and can therefore be used as food supplement for both human and in animal feed. The result of this researchcould therefore provide lead for future investigation.
\end{abstract}

Keywords: Moringa oleifera; Maceration; LCMS-QToF; X-ray Fluorescence

\subsection{INTRODUCTION}

Moringa oleifera is an important fast-growing tree belonging to the family of Moringaceae and is commonly regarded as miracle tree due to its nutritional and medicinal benefits (Al_husnan \& Alkahtani, 2016). There are thirteen (13) species of this tropical plant which widely grown in tropical many parts of Asia and sub-Sahara Africa (Chen et al., 2017). Almost every part of Moringa oleifera can be used for food and as forage for livestock (Al husnan \& Alkahtani, 2016). The leaves can be eaten fresh cooked or stored as dried powder for several months the pods, when young can be cooked; eaten like beans. Its oil and micronutrients have been reported to contain antitumour, antiepileptic, antidiuretic, anti-inflammatory and venomous bite characters (Anwar \& Bhanger, 2003). 
These functional properties of different parts of Moringa oleifera are due to the presence of bioactive compounds and mineral elements. The Moringa oleifera husk for example is generally known for its numerous benefits such as in the purification of water with their medicinal and nutritional properties (Ijarotimi et al., 2017).However, several investigations have demonstrated that the bioactive and mineral elements from Moringa oleifera husk makes them a good candidate for nutraceutical applications (Salaheldeen et al., 2015). These bioactive and elemental constituents are responsible for the different biological functions such as antioxidant and anti-microbial as reported by Salaheldeen et al. (2015).

Furthermore, as a result of the functional properties of Moringa oleifera husk to human health, it is imperative to determine an appropriate standard method characterization to produce high quality bio-products from this commodity crop. The minerals and chemical composition of Moringa oleifera plants and their bioavailability have been a subject of tremendous research but there are limited studies on the profiling and valorization of husk waste using state-of-the-art analytical techniques. The objective of this study was to investigate the mineral and chemical components of the Moringa oleifera husk extracts using the Liquid Chromatography equipped with an electrospray time-of-flight mass spectrometer (LCMS-QToF) and X-ray fluorescence analysis.

\subsection{Materials Preparation}

\subsection{MATERIALS AND METHODS}

The Moringa oleifera was carefully separated from its seeds was pulverized using a Grindomix grinder (GM-200 model, Germany). The powdered Moringa oleifera husk (without seeds) were stored in an airtight container. Ten grams (10 g) of the sample was sequentially macerated in $100 \mathrm{~mL}$ of analytical grade ethanol. The macerate was first filtered through a Whatmann No. 1 filter paper and concentrated using a rotary evaporator (R-200 model, Germany). The extracts were thereafter stored at $-20{ }^{\circ} \mathrm{C}$ until analysis.

\subsection{LCMS-QToF Analysis}

The chemical profiling was conducted using a Liquid Chromatography equipped with an electrospray time-of-flight mass spectrometer (LCMS-QToF) detectors. The chromatographic separation was carried out using ACQUITY UPLCHSS $\mathrm{T}_{3}$-column ( $2.1 \times 100 \mathrm{~mm}$, particle size $1.8 \mu \mathrm{m}$ ) (Waters, USA). The mobile phase was; A, $0.1 \%$ formic acid; B $0.1 \%$ formic acid in acetonitrile (Olalere et al., 2018). The mass spectrometer was equipped with an electrospray ionization source operated in positive ion mode. The extracts were tentatively characterized in accordance with their ions' mass fragmentation.

\subsection{XRF Analysis}

The X-ray fluorescence analysis was employed to simultaneously measure and analyze the microelements and trace elements in the Moringa oleifera husk extracts. The atoms in the sample were excited using the monochromatic radiation emission from the X-ray tubing in an S8 Tiger Quant express analyzer. The analysis was conducted with the aid of a three-axial geometric configurations in order to mitigate the effect of noise emanating from polarization (Olalere et al.,2018). 


\subsection{RESULTS AND DISCUSSION}

\subsection{Identification of Bioactive Compounds in the Extracts}

The bioactive composition of the Moringa oleifera husk extracts were identified and confirmed based on the mass-fragmentation and spectra chromatographs as illustrated in Figure 1. A total of 493 metabolites were identified and obtained by electron spray ionization mass spectrometry (ESI-MS/MS) with the corresponding confirmation of their spectra, retention times, molecular and ionic fragmentation of each chemical components. A combination of these bioactive compounds is largely responsible for the numerous medicinal and nutritional characteristics of Moringa oleifera husk as reported by Ijarotimi et al. (2017).

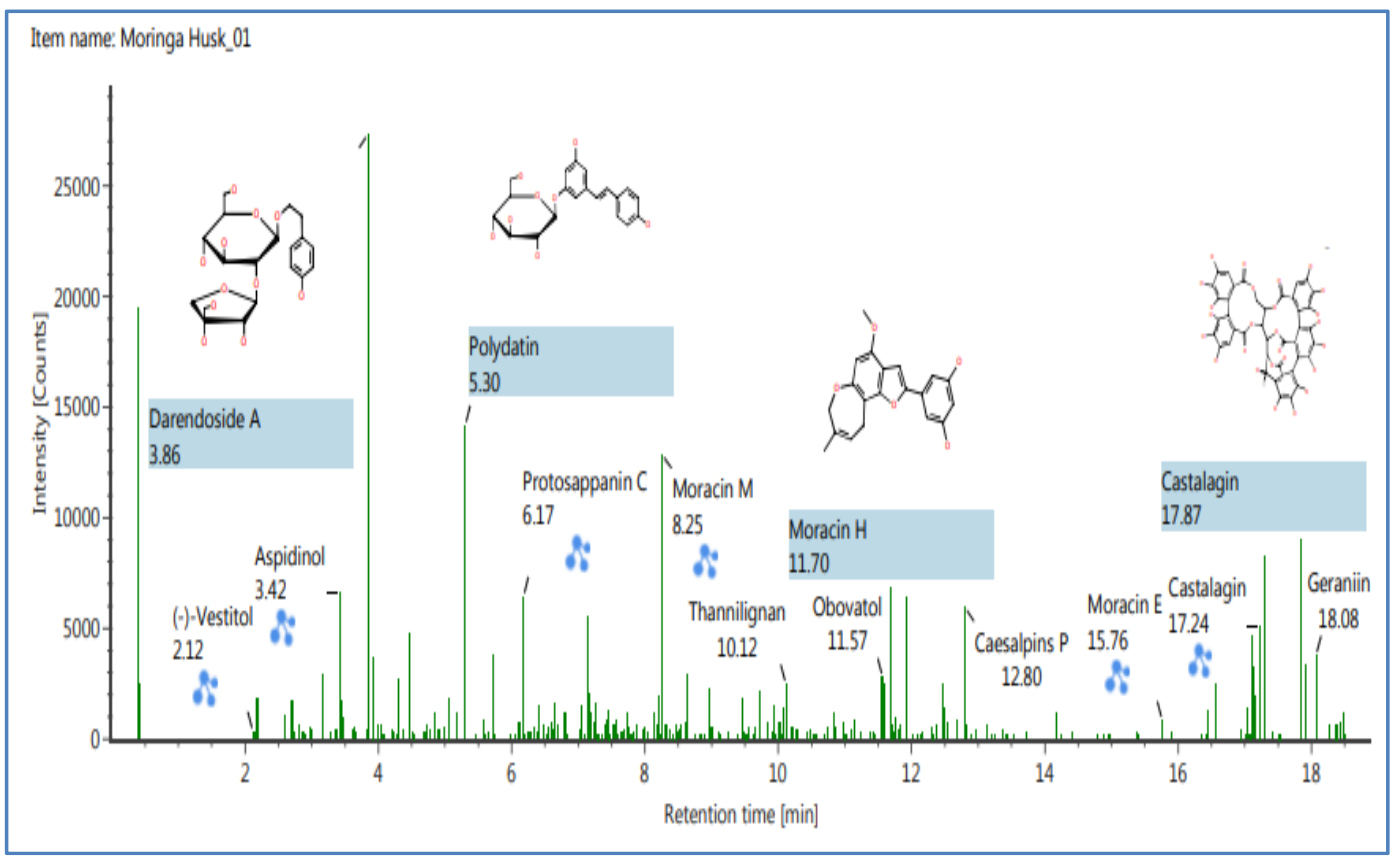

Figure 1: Chemical profiling of Moringa oleifera husk extracts by LC-MS-Q-ToF

\subsection{Estimation of mineral element concentration}

The XRF-mineral analysis was conducted to determine the micronutrients composition in the $M$. oleifera husk with the results obtained showed remarkable higher concentration in $\mathrm{Ca}$, and $\mathrm{K}$. Moreover, a weaker concentration was obtained for S, P, $\mathrm{Cl}, \mathrm{Fe}$ and $\mathrm{Mg}$, as presented in Table 1 . However, the concentration of trace elements $(\mathrm{Cu}, \mathrm{Si}, \mathrm{Mo}$, and $\mathrm{Sr})$ were found to be negligible which indicated the edibility and nontoxic characteristics of the $M$. oleifera husk extracts as food fortifants as reported by Oyeyinka \& Oyeyinka (2018). 
Table 1: Mineral content of $M$. oleifera husk extracts obtained by X-ray fluorescence Banalysis

\begin{tabular}{llc}
\hline No & Parameter & Result (\%)* \\
\hline 1 & Calcium (Ca) & $39.40 \pm 0.21$ \\
2 & Potassium (K) & $38.29 \pm 0.37$ \\
3 & Sulphur (S) & $8.29 \pm 0.09$ \\
4 & Phosphorus (P) & $4.98 \pm 0.23$ \\
5 & Chlorine (Cl) & $2.81 \pm 0.11$ \\
6 & Iron (Fe) & $2.78 \pm 0.43$ \\
7 & Magnessium (Mg) & $1.99 \pm 0.12$ \\
8 & Zinc (Zn) & $0.41 \pm 0.33$ \\
9 & Copper (Cu) & $0.33 \pm 0.17$ \\
10 & Silicon (Si) & $0.29 \pm 0.42$ \\
11 & Molybdenum (Mo) & 0.15 \\
12 & Strontium (Sr) & 0.14 \\
\hline
\end{tabular}

*Average \pm Standard Deviation

\subsection{CONCLUSIONS}

The aim of this work is to access the elemental and phenolic profile in M. oleifera husk extracts using LC-QToF/MS and X-ray fluorescence analysers. The results of the mineral element and phytochemical screening showed the presence of 493 secondary metabolites, 7 micronutrients and 4 trace elements. The presence of lower concentration in the trace elements indicated that edibility of the husk extracts and its potential use in the food and/or pharmaceutical industries. Moreover, the cumulative bioactivities of these chemical and elemental constituents are responsible for the numerous therapeutic functions of Moringa oleifera husk as reported by many researchers. The result described in this investigation can therefore be used for further investigation into the other nutraceutical applications of Moringa oleifera husk extracts.

\section{ACKNOWLEDGEMENT}

This work was partially supported by International Islamic University Malaysia, (RIGS 16-075-0239) from Ministry of Higher Education of Malaysia.

\section{REFERENCES}

Al_husnan, L. A. andAlkahtani, M. D. F. (2016). Impact of moringa aqueous extract on 247-250. pathogenic bacteria and fungi in vitro. Annals of Agricultural Science, 61(2),

Anwar, F. and M.I. Bhanger, (2003). Analytical characterization of Moringa oleifera seed oil grown in temperate regions of Pakistan. Journal of Agriculture and Food Chemistry, 51, 6558-6563

Chen, C., Zhang, B., Huang, Q., Fu, X. and Liu, R. H. (2017). Microwave-assisted extraction of polysaccharides from Moringa oleifera Lam. leaves: Characterization and hypoglycemic activity. Industrial Crops and Products, $100,1-11$.

Ijarotimi, O. S., Adeoti, O. A. andAriyo, O. (2017). Comparative study on nutrient composition, phytochemical, and functional characteristics of raw, germinated, and fermented Moringa oleifera seed flour. Food Science \& Nutrition, 1(6), 452-463 
Salaheldeen, M., Aroua, M. K., Mariod, A. A., Chenge, S. F., Abdelrahman, M. A. and Atabani, A. E. (2015). Physicochemical characterization and thermal behaviour of biodiesel and biodiesel-diesel blends derived from crude Moringa peregrina seed oil. Energy Conversion and Management, 92, 535-542

Oyeyinka, A. T. and Oyeyinka, S. A. (2018). Moringa oleifera as a food fortificant:

Recent trends and prospects. Journal of the Saudi Society of Agricultural Sciences, 17, 127-137.

Olalere, O.A., Abdurahman, N.H., Alara, O. R., and Habeeb,O.A. (2018). Chemical fingerprinting of biologically active compounds and morphological transformationduring microwave reflux extraction of black pepper. Chemical Data Collection, 17-18, 339-344.

Olalere, O.A, Abdurahman, N. H., Yunus, R.M., and Alara, O. R. (2017). The effects of microwave heating on the extraction yield and elemental composition of black and white pepper (Piper nigrum) extracts. Journal of Chemical Engineering \& Industrial Biotechnology, V2, 59-67. 